Originalien

Psychotherapeut 2021 · 66:247-257 https://doi.org/10.1007/s00278-020-00482-2 Angenommen: 7. Dezember 2020 Online publiziert: 6 . Januar 2021

(c) Der/die Autor(en) 2021
Im neuen Psychotherapiestudium an den psychologischen Instituten der Universitäten wird der praxisorientierte und kompetenzbasierte Unterricht in den anerkannten Methoden und Verfahren eine zentrale Rolle spielen. Hier wird der frühen, longitudinalen und spiralförmigen Integration handlungsorientierter Lehrformen große Bedeutung zukommen. Allerdings stehen bislang keine Konzeptionen entsprechender Curricula zur Verfügung. Das im Folgenden vorgestellte DYNAMIKCurriculum soll dazu beitragen, diese Lücke zu schließen.

\section{Einleitung}

\section{Säulen der Psychotherapie- ausbildung und notwendige Kompetenzorientierung}

Grundlegende Säulen der derzeitigen Ausbildung psychologischer Psychotherapeuten ${ }^{1}$ sind in der Wissensvermittlung theoretischer Inhalte, der praktischen

\footnotetext{
1 Der besseren Lesbarkeit halber umfassen Funktionsbezeichnungen in der männlichen Form stets alle Genera.
}

C. Nikendei' $\cdot$ U. Dinger ${ }^{1} \cdot$ J. Dück' $\cdot$ J. C. Ehrenthal ${ }^{2,3} \cdot$ T. Storck $^{4} \cdot$ S. Taubner ${ }^{5}$. H.-C. Friederich ${ }^{1} \cdot$ H. Schauenburg' $\cdot$ J.-H. Schultz ${ }^{1} \cdot$ I. Montan ${ }^{1}$

'Zentrum für Psychosoziale Medizin, Klinik für Allgemeine Innere Medizin und Psychosomatik, Universitätsklinikum Heidelberg, Heidelberg, Deutschland

${ }^{2}$ Department Psychologie, Universität Köln, Köln, Deutschland

${ }^{3}$ Institut für Medizinische Psychologie, Universität Heidelberg, Heidelberg, Deutschland

${ }^{4}$ Psychologische Hochschule Berlin, Berlin, Deutschland

${ }^{5}$ Institut für Psychosoziale Prävention, Universität Heidelberg, Heidelberg, Deutschland

\title{
Konzeption des longitudinalen DYNAMIK-Curriculums
}

\section{Vermittlung psychodynamischer Interventionskompetenzen in der Ausbildung psychologischer Psychotherapeuten}

Tätigkeit in psychiatrischen und psychosomatischen Kliniken (praktische Tätigkeit PT1 und PT2), der Selbsterfahrung, der praktischen Ausbildung im Sinne der selbstständigen Durchführung ambulanter Psychotherapien sowie deren Supervision durch erfahrene Psychotherapeuten und in der "freien Spitze“ zu sehen (nach Interessenneigung gewählte Ausbildungseinheiten; Strauß et al. 2009). Dabei wird die selbstständige, unter begleitender Supervision durchgeführte Behandlung ambulanter Patienten vonseiten der in Ausbildung befindlichen Therapeuten für die Entwicklung eigener therapeutischer Kompetenzen als besonders hilfreich erlebt (Strauß et al. 2009). Die erfahrungsbasierte, praxisorientierte Vermittlung der in der Therapie angewandten Interventionen in Form von speziell konzipierten Trainings oder Übungseinheiten spielt formell in der aktuellen Ausbildung werdender Psychotherapeuten hingegen lediglich eine marginale Rolle.

Durch die Einführung des neuen "Psychotherapiestudiums“ zum Herbst 2020 einerseits sowie die formulierten Wünsche und Erwartungen bisheriger Ausbildungsteilnehmer andererseits (Nikendei et al. 2018), ist es notwendig, erfahrungsbasierte und kompetenzorientierte Lehrinhalte und Prüfungsformate in die derzeitige Ausbildung und das zukünftige „Psychotherapiestudium“ mit nachfolgender Weiterbildung $\mathrm{zu}$ integrieren. In der neuen Approbationsordnung ist der Einsatz von standardisierten Patienten ([Laien-]Schauspielern, die Patienten in einem simulierten Setting portraitieren; Barrows 1968) sowohl im Rahmen des Studiums in Form praktischer Übungen und Seminare ( $\$$ ) als auch im Rahmen der Approbationsprüfung in Form von ,anwendungsorientierten Parcoursprüfungen“ (Unterabschnitt 3, $\$ \$ 48,49$ ) vorgesehen. Entsprechend bestehen ein dringender Bedarf an konzeptuellen Entwicklungen zur Vermittlung und zur Prüfung praktischer psychotherapeutischer Kompetenzen sowie die Notwendigkeit, diese wissenschaftlich $\mathrm{zu}$ evaluieren.

\section{Interventionskompetenzen in der psychodynamischen Psychotherapie}

Die nachhaltige Wirksamkeit psychodynamischer Psychotherapie ist in zahlreichen Übersichtsarbeiten und Metaanalysen belegt (Steinert et al. 2017). Eine 
tragfähige Beziehung zwischen dem $\mathrm{Pa}$ tienten und dem Therapeuten trägt als zentraler Faktor entscheidend zum Erfolg der psychotherapeutischen Behandlung bei (Horvath et al. 2011), sodass allgemeinen therapeutischen Kompetenzen zum Aufbau, zur Aufrechterhaltung bzw. ggf. zur „Reparatur“ (Safran et al. 2011) einer tragfähigen therapeutischen Beziehung eine besondere Bedeutung zukommt. Innerhalb dieser therapeutischen Arbeitsbeziehung können durch spezifische Interventionen Prozesse zur Förderung der Mentalisierungsfähigkeit (Fischer-Kern et al. 2015), der Einsicht von Patienten (Jennissen et al. 2018) und deren interpersonellen Fähigkeiten (Crits-Christoph et al. 2010) in Gang gesetzt werden. Dies könnte - trotz bisher fehlender Evidenz - bedeuten, dass die sichere und kompetente Anwendung psychodynamischer Interventionen zur Verbesserung der therapeutischen Beziehung und damit auch zum Erfolg der Therapie beiträgt.

Internationale Untersuchungen von Novizen, die am Beginn ihrer Tätigkeit als Behandler stehen, haben ergeben, dass diese sich sowohl um ihre technischen Fertigkeiten sorgen als auch darum, inwieweit sie sich ihrer eigenen inneren Reaktionen bewusst sind, um mit diesen im therapeutischen Prozess adäquat umzugehen (Hill et al. 2007). Dies spiegelt sich u.a. in der Beobachtung wider, dass sich Therapeuten in Ausbildung zu Beginn ihrer selbstständigen Behandlung ambulanter Patienten als stark herausgefordert und unter Stress erleben (Taubner et al. 2010). Vor diesem Hintergrund ist es nicht verwunderlich, dass sowohl internationale Befragungen als auch Studien aus dem deutschsprachigen Raum (Nikendei et al. 2018) zeigen, dass Praxiselemente und praktische Übungen als besonders wertvoll wahrgenommen werden sowie deren praxis- und handlungsorientierte Vermittlung gefordert wird.
Vermittlung psychotherapeutischer Kompetenzen in der Ausbildung psychodynamischer Psychotherapie

Psychodynamische Interventionen stehen im Spannungsfeld zwischen Beziehungsaufbau und Vermittlung therapeutischer Veränderungsprozesse. Trotz dieser bedeutsamen Stellung beschäftigen sich lediglich wenige Studien, die therapeutische Interventionskompetenzen im Rahmen spezifischer Trainingsprogramme fokussieren, mit deren Wirksamkeit z.B. in einer signifikanten Zunahme der Häufigkeit spezifischer psychodynamischer Techniken in der Patientenbehandlung. Hierzu zählen z.B. die Identifizierung wiederkehrender Erlebens- und Verhaltensmuster sowie die Fokussierung auf Träume und Fantasien (Hill und Lent 2006). Im Rahmen dieser Programme, wie z.B. dem „Helping Skills Training“ (Hill und Lent 2006), werden für alle Therapieschulen relevante Interventionen („common factors“), wie die Technik des Spiegelns oder das Stellen offener Fragen, in PeerRollenspielen geübt. Der Einsatz von standardisierten Patienten, der in der Ausbildung von angehenden Ärzten nicht mehr wegzudenken ist, stellte in der psychotherapeutischen Ausbildung bislang noch eine Seltenheit dar. Nach der neuen Approbationsordnung vom März 2020 ist dieser für das Masterstudium und die Approbationsprüfung zwar explizit vorgesehen, allerdings sind den Autoren keine Konzeptionen entsprechender longitudinaler Curricula bekannt. Das DYNAMIK-Curriculum soll dazu beitragen, diese Lücke zu schließen.

\section{Studien zur Arbeit mit Peer- Rollenspielen und standardisierten Patienten}

Im deutschsprachigen Raum existieren nur wenige Untersuchungen zur Vermittlung psychodynamischer Kompetenzen mithilfe von Peer-Rollenspielen und standardisierten Patienten. Beim Peer-Rollenspiel, also dem Rollenspiel innerhalb einer Gruppe von Ausbildungsteilnehmern, nehmen einzelne
Gruppenmitglieder klassischerweise die Rolle des Patienten, Behandlers oder des Beobachters ein. Dieses Setting ermöglicht es den Therapeuten in Ausbildung, im Rahmen der Peer-Rollenspiele auch die Innensicht des Patienten zu erleben (Bosse et al. 2012). Ehrenthal (2019) untersuchte den subjektiven Kompetenzzuwachs von Masterstudenten der Psychologie in einem erfahrungsbasierten, praxisorientierten Seminar zur psychodynamischen Kurzzeittherapie unter dem Einbezug von Peer-Rollenspielen. Es zeigten sich ein signifikanter subjektiver Kompetenzgewinn sowie eine hohe Akzeptanz erfahrungsbasierter Lehrformate.

Rollenspiele in Kombination mit deren Aufzeichnung per Video und anschließender (wertschätzender) Evaluation in der Gruppe sind ebenfalls zentrales Element des allianzfokussierten Trainings (Eubanks-Carter et al. 2015), das aktuell in Pilotprojekten auch in Deutschland implementiert wird (Gumz 2020; Gumz et al. 2018). Es erweist sich als wirksam darin, die therapeutischen Skills Selbstwahrnehmung, Affektregulation und Beziehungskompetenz $\mathrm{zu}$ schulen, um diese im Kontext von Brüchen in der therapeutischen Beziehung so einzusetzen, dass Krisen im Therapieprozess konstruktiv genutzt und mit dem Patienten gemeinsam bewältigt werden können (Safran et al. 2014).

Der Begriff standardisierte Patienten bezeichnet (simulierende oder reale) „Schauspiel“-Patienten, die dafür trainiert werden, ihre erlernte (oder tatsächliche) Erkrankung in einer gleichbleibenden Art und Weise für Lehrzwecke im Kontext von Rollenspielen zu präsentieren (Barrows 1968). Standardisierte Patienten werden als sehr realistisch wahrgenommen und sind darin geschult, ein professionelles Feedback zu ihrer Wahrnehmung der Interaktion mit dem Behandler zu geben (Schultz et al. 2018). Im Rahmen einer Präpost-Studie untersuchten Partschefeld et al. (2013) 29 Ausbildungsteilnehmer bei einem Training von Gesprächskompetenzen nach Hill (2014) unter dem Einbezug von standardisierten Patienten. Die therapeutischen Fertigkeiten wurden sowohl aus der Perspektive der 
Selbst- als auch der Fremdbeurteilung nach der Absolvierung des Trainings als signifikant besser eingeschätzt. Darüber hinaus verbesserten sich empathische Fähigkeiten, Selbstwirksamkeitserleben und die therapeutische Allianz. Nikendei et al. (2019) untersuchten bei 20 Ausbildungsteilnehmern eines psychodynamischen Ausbildungsinstituts in einem Prä-post-Design den Einsatz von Rollenspielen mit Peers und standardisierten Patienten zum Erwerb psychodynamischer Interventionskompetenzen in Bezug auf 5 Interventionen (Klarifizieren, Spiegeln, Konfrontieren, Antworten und Deuten; Nikendei et al. 2019). Klarifizierende Interventionen wurden im Sinne von Transfereffekten in den Psychotherapiesitzungen häufiger und auch in der Fremdbeurteilung kompetenter eingesetzt. Der subjektive Lernerfolg korrelierte insbesondere mit dem positiven Erleben der Übung mit den standardisierten Patienten, dem als hilfreich erlebten Dozentenfeedback und einem antizipierten Transfer in den therapeutischen Alltag (Ehrenthal et al. 2020).

Der Einsatz standardisierter Patienten nicht nur in der praktischen Vermittlung psychotherapeutischer Kompetenzen, sondern auch in deren Prüfung - angelehnt an die in der Medizin etablierten „objective structured clinical examinations" (OSCE) - wird gegenwärtig evaluiert. Er erweist sich als geeignete Prüfungsmethode, die zwar mit einem hohem Angstniveau aufseiten der Prüflinge verknüpft ist, aber dennoch als realistische, valide und faire Prüfungsmethode wahrgenommen und als positive Lernerfahrung beurteilt wird (Gumzetal. 2020; Kühne et al. 2020; Yap et al. 2012; Sheen et al. 2015). Wesentlich scheint die Fähigkeit der standardisierten Patienten zu sein, eine als authentisch erlebte klinische Situation herzustellen (Melluish et al. 2007).

\section{Zielsetzung der vorliegenden Arbeit}

Ziel der vorliegenden Arbeit ist die Darstellung der Entwicklung und Konzeption des longitudinalen Curriculums zur Vermittlung psychodynamischer Inter-

Psychotherapeut 2021 -66:247-257 https://doi.org/10.1007/s00278-020-00482-2

(c) Der/die Autor(en) 2021

C. Nikendei - U. Dinger · J. Dück · J. C. Ehrenthal · T. Storck · S. Taubner · H.-C. Friederich · H. Schauenburg $\cdot$ J.-H. Schultz $\cdot$ I. Montan

\section{Konzeption des longitudinalen DYNAMIK-Curriculums. Vermittlung psychodynamischer Interventionskompetenzen in der Ausbildung psychologischer Psychotherapeuten}

\section{Zusammenfassung}

Hintergrund. Durch die Einführung des neuen "Psychotherapiestudiums" zum Herbst 2020 und die Wünsche bisheriger Ausbildungsteilnehmer an psychotherapeutischen Ausbildungsinstituten ist die Notwendigkeit gegeben, erfahrungsbasierte und kompetenzorientierte Lehre in das zukünftige Studium und die nachfolgende Weiterbildung werdender Psychotherapeuten zu integrieren.

Material und Methode. Orientiert am Kern-Zyklus, einem bedeutenden Modell zur Curriculumsentwicklung, werden die Entwicklung, Konzeptionalisierung und Implementierung eines longitudinalen Curriculums zur Vermittlung psychodynamischer Interventionskompetenzen (DYNAMIKCurriculum) in die derzeitige Ausbildung zum psychologischen Psychotherapeuten vorgestellt.

Ergebnisse. Es wurde ein longitudinales Curriculum mit 8 Modulen zur Vermittlung allgemeiner und spezifischer psychodynamischer Interventionskompetenzen mithilfe von Peer-Rollenspielen und standardisierten Patienten entwickelt. Dieses stellt seit Mitte 2019 einen integralen Bestandteil der Ausbildung zum tiefenpsychologisch fundierten Psychotherapeuten am Heidelberger Institut für Psychotherapie (HIP) dar.

Schlussfolgerung. Das DYNAMIK-Curriculum ist der erste wichtige Schritt in Richtung der longitudinalen erfahrungsorientieren, praxisnahen Kompetenzorientierung in der derzeitigen Ausbildung und dem zukünftigen Studium mit nachfolgender Weiterbildung von psychologischen Psychotherapeuten. Es resultiert die Notwendigkeit zur Wirksamkeitsüberprüfung solcher Curricula und zur praktischen Prüfung von Interventionskompetenzen.

Schlüsselwörter Psychotherapeutische Ausbildung . Psychodynamische Interventionen . Kompetenzbasiertes Lernen · Standardisierte Patienten · Peer-Rollenspiel

\section{DYNAMIC-A longitudinal curricular concept. Teaching psychodynamic intervention skills during postgraduate psychotherapy training}

\section{Abstract}

Background. In Germany, the launch of the new university "psychotherapy degree program" in autumn 2020 and the wishes of current and future psychotherapists at psychotherapy training institutes have made it necessary to integrate experience-based and competence-oriented teaching into future university and postgraduate curricula. Material and methods. Following Kern's sixstep approach to curriculum development, the conceptualization, development, and implementation of a longitudinal curriculum for teaching psychodynamic intervention skills (DYNAMIK curriculum) to future psychological psychotherapists is showcased. Results. A longitudinal curriculum with eight modules using peer role play and standardized patients was developed to teach general and specific psychodynamic inter- vention skills. The DYNAMIK curriculum was incorporated into the current postgraduate, depth psychology-focused, psychotherapist training program.

Conclusion. The DYNAMIK curriculum is an important first step towards longitudinal experience-based and competence-oriented teaching in current postgraduate and future university degree program curricula followed by postgraduate psychotherapist training. Hence, the effectiveness of such curricula and intervention competencies need to be reviewed.

Keywords

Psychotherapy training - Psychodynamic interventions . Competency based training . Standardized patients . Peer role play 
ventionskompetenzen („DYNAMIK“) anhand des Modells zur Curriculumsentwicklung nach Kern (Kern-Zyklus; Kern et al. 1998). Die vorgeschlagene Konzeption eignet sich zur didaktischen Gestaltung sowohl der Ausbildung psychologischer Psychotherapeuten als auch des zukünftigen „Psychotherapiestudiums" mit nachfolgender Weiterbildung.

\section{Methoden}

\section{Setting}

Die Implementierung des DYNAMIKCurriculums erfolgt derzeit am Heidelberger Institut für Psychotherapie (HIP; Schauenburg et al. 2019). Das HIP ist eine staatlich anerkannte Ausbildungsstätte, die an die Klinik für Allgemeine Innere Medizin und Psychosomatik am Zentrum für Psychosoziale Medizin des Universitätsklinikums Heidelberg angegliedert ist. Gegründet wurde das HIP 2010 und bietet seitdem jedes Jahr 20 Ausbildungsteilnehmern eine 5-jährige Weiterbildung zum psychologischen Psychotherapeuten mit psychodynamischem Schwerpunkt (tiefenpsychologisch fundierte Psychotherapie). Seit dem Jahr 2020 gibt es zudem einen systemischen Ausbildungszweig. Es existiert ein den Richtlinien entsprechendes Ausbildungscurriculum mit Vorlesungen, Seminaren, Theorie-Praxis-Gruppen und freier Spitze. Derzeit werden von den Ausbildungsteilnehmern am HIP über 500 aktiv laufende ambulante Psychotherapien unter Supervision durchgeführt (Stand: 05.06.2020).

\section{Methodisches Vorgehen: Curriculumsentwicklung nach Kern}

Die Konzeption des kompetenzbasierten DYNAMIK-Curriculums folgte dem Zyklus der Curriculumsentwicklung nach Kern et al. (1998). Die Steuerungsgruppe, bestehend aus 2 ärztlichen und 2 psychologischen wissenschaftlichen Mitarbeitern (C.N., U.D., J.D., I.M.), arbeitet/ arbeitete hierfür eng mit dem Leitungsgremium des HIP zusammen. Sie verantwortet die Definition der zu vermittelnden Kompetenzbereiche, die Definition der einzelnen Lernziele, die Entwicklung desStundenplans, die Auswahl der didaktischen Methoden sowie die Koordination der Implementierung und Evaluation des DYNAMIK-Curriculums. Für die inhaltliche Ausgestaltung der einzelnen Module wurden weitere klinische Fachexperten als Kooperationspartner einbezogen (ST, TS, JE, HS).

Nachfolgend werden im Ergebnisteil die Resultate der einzelnen Entwicklungsschritte des Curriculums entsprechend dem Kern-Zyklus dargestellt, der sich in die Teilschritte A-F gliedert:

A. Problemdefinition und allgemeine Bedarfsanalyse,

B. Bedarfsanalyse der Zielgruppen,

C. Definition der Lern- und korrespondierenden Prüfungsziele,

D. Lehrstrategien,

E. Implementierung,

F. Evaluation.

\section{Ergebnisse und Umsetzung}

\section{A Problemdefinition und allgemeine Bedarfsanalyse}

Die Problemdefinition der unzureichenden kompetenz- und praxisorientierten Vermittlung psychodynamischer Interventionen im curricularen Teil der Ausbildung psychodynamischer Psychotherapeuten (Istzustand) wurde durch eine detaillierte Literaturrecherche in den $\mathrm{Da}$ tenbanken PsycINFO, PubMed, Psyndex und Google Scholar mit den Suchbegriffen "psychotherapy training“, ,roleplay“, „standardized patients“, „psychodynamic interventions" inhaltlich untermauert. Aus den in der Einleitung vorgestellten Forschungsarbeiten sowie aus den durch die Approbationsordnung vom März 2020 gestellten Anforderungen ist abzuleiten, dass allgemein ein dringender Bedarf an erfahrungsbasierten, kompetenzorientierten Lehrformaten zur Vermittlung psychodynamischer Interventionskompetenzen besteht.

\section{B Bedarfsanalyse der Zielgruppe}

Als Bedarfsanalyse der spezifischen Zielgruppe wurde eine freiwillige Befragung zu den Erwartungen und Wünschen bei 12 von 20 Ausbildungsteilnehmern zu
Beginn ihrer tiefenpsychologisch-fundierten Psychotherapie durchgeführt. Limitierend ist zu benennen, dass Dozenten und Lehrverantwortliche nicht in die Befragung eingeschlossen wurden. Die inhaltsanalytische qualitative Auswertung der halbstandardisierten Interviews ergab, dass sich die angehenden tiefenpsychologischen Psychotherapeuten praktische therapeutische Übungen in einem geschützten Rahmen mit einem besonderen Fokus auf die Patient-Therapeut-Beziehung wünschen (Nikendei et al. 2018). Damit war die allgemeine Bedarfsanalyse für die Zielgruppe bestätigt, und der Soll-Ist-Zustand entsprechend Kern et al. (1998) konnte abgeleitet werden.

\section{Definition der Lern- und Prüfungsziele}

Als übergeordnetes Lernziel (Sollzustand) wurde definiert, dass "der Absolvent des longitudinalen DYNAMIKCurriculums in der Lage ist, psychodynamische Interventionen, angepasst an die strukturellen Möglichkeiten und vordringlichen psychodynamischen Konfliktthemen des Patienten (Operationalisierte Psychodynamische Diagnostik [OPD]; Schauenburg et al. 2020), unter Reflexion der therapeutischen Beziehung und dem persönlichen Gegenübertragungserleben kompetent einzusetzen“. Somit soll er dazu befähigt werden, fallbezogene Reflexionsprozesse im Rahmen der Supervision ambulanter Therapien kompetenzorientiert $\mathrm{zu}$ ergänzen. Als thematische Bereiche wurden 8 Module festgelegt, innerhalb derer korrespondierende Lernziele von der Steuerungsgruppe zusammen mit den vorgesehenen Dozenten der Module expertenbasiert definiert wurden, um die dem thematischen Bereich zugehörigen psychodynamischen Interventionskompetenzen entsprechend $\mathrm{zu}$ trainieren. Auch bei originär transtheoretischen Ansätzen, wie etwa dem Umgang mit Brüchen der therapeutischen Arbeitsbeziehung, wurden die zugehörigen Interventionsstrategien in psychodynamische Modelle von konflikt- und strukturbezogenen Interventionsstrategien eingeordnet. Es wurde außerdem 
Tab. 1 Modulstruktur, Modulthemen und Lernziele des DYNAMIK-Curriculums

\begin{tabular}{l|l|l|l}
\hline $\begin{array}{l}\text { Ausbildungsjahrgang/ } \\
\text { Thema }\end{array}$ & Modul & Interventionen & Lernziele/Kompetenzen (Beispiel) \\
\hline $\begin{array}{l}\text { Jahr 1/HJ } 1 \text { bis } \\
\text { Jahr } 4 / \text { HJ } 4\end{array}$ & I-VIII & $\begin{array}{l}\text { Allgemeine Interventi- } \\
\text { onskompetenzen }\end{array}$ & $\begin{array}{l}\text { Der Therapeut formuliert die Intervention an einer stimmigen Stelle im therapeuti- } \\
\text { schen Gespräch klar, deutlich und flüssig, höflich und taktvoll, in einer Weise, die für } \\
\text { den Patienten in seiner „Welt" und Sprache inhaltlich gut nachvollziehbar ist }\end{array}$ \\
\hline $\begin{array}{l}\text { Jahr 1/HJ } 1 \\
\text { Basisinterventionen im } \\
\text { Kontext von Übertra- } \\
\text { gung und Gegenüber- }\end{array}$ & $\mathrm{I}$ & $\begin{array}{l}\text { Spiegeln } \\
\text { Klären } \\
\text { Konfrontieren }\end{array}$ & $\begin{array}{l}\text { Keispiel Konfrontieren: Der Therapeut erkennt und benennt Widersprüche, indem er } \\
\text { diskrepantes Material gegenüberstellt, das der Patient offenbar nicht als widersprüch- } \\
\text { lich wahrnimmt oder gar nicht wahrnimmt }\end{array}$ \\
\hline
\end{tabular}

gung und Gegenübertragung

\begin{abstract}
Jahr $1 /$ HJ 2
Strukturbezogene

Interventionen
\end{abstract}

Sich „hinter den Patienten stellen"

Sich "neben den Patienten stellen"

Sich „dem Patienten gegenüberstellen"

\section{Jahr 2/HJ 1}

Mentalisierungsbezogene Interventionen

\section{Jahr 2/HJ 2}

Abwehr- und Widerstandsdeutung

\section{Jahr 3/HJ 1}

Übertragungsdeutung

\section{Jahr 3/HJ 2}

Umgang mit Brüchen

in der Psychotherapie („rupture repair")

Jahr 4/HJ 1

Arbeit an dysfunktionalen Beziehungsmustern

\section{Jahr 4/HJ 2}

VIII

Arbeit am therapeutischen Fokus

\section{MBT-Grundhaltung}

Affekte fokussieren und empathisch validieren

Alternative Perspektiven eröffnen

IV Abwehrdeutung

Widerstandsdeutung

V Übertragungsdeutung
Umgang und Reparatur von Brüchen in der therapeutischen Arbeitsbeziehung

Wahrnehmung und Differenzierung dysfunktionaler Beziehungsmuster

Formulierung eines therapeutischen Fokus

Halten eines therapeutischen Fokus

Der Therapeut erkennt strukturelle Defizite des Patienten, die bei dem aktuell in der Therapie behandelten Thema relevant sind; er ist in der Lage, eine entsprechend hilfreiche strukturbezogene therapeutische Position einzunehmen und diese adäquat zu verbalisieren

Der Therapeut zeigt dem Patienten gegenüber eine empathisch-wertschätzende, nicht-wissend neugierige, aktiv-balancierende und das Mentalisieren verstärkende Grundhaltung

Der Therapeut erkennt verbale oder behaviorale Formen der Abwehr oder des Widerstands beim Patienten, er kann die Deutung an einem Dringlichkeitspunkt anbringen/ verbalisieren/platzieren; er gibt die Abwehr- oder/Widerstandsdeutung in einer behutsamen und für den Patienten gesichtswahrenden Art und Weise

Der Therapeut kann die Wiederholung (Reinszenierung) von für den Patienten typischen Beziehungsgestaltungsmustern in der therapeutischen Beziehung erkennen und benennen; er gibt die Deutung an einem Dringlichkeitspunkt; er differenziert zwischen der ursprünglichen und der aktuellen Beziehungserfahrung

Der Therapeut kann einen mit Rückzug einhergehenden Bruch identifizieren, diesen benennen und angemessen auf den Rückzug reagieren

Der Therapeut ist in der Lage, dysfunktionale Muster in Beziehungsepisoden des Patienten unter Berücksichtigung des eigenen Erlebens in der therapeutischen Beziehung zu erkennen und diese auf passenden Interventionsebenen zu adressieren

Der Therapeut ist in der Lage, eine geeignete Fokusformulierung zu entwickeln und mit dem Patienten zu besprechen; er kann im Verlauf der Therapie die therapeutische Arbeit nach dem Fokus ausrichten; er kann zwischen freier Gesprächsentwicklung und Fokusbezug balancieren ein Modulelement zur Mentalisierungsfähigkeit integriert, da die zentralen Beziehungskonflikte implizieren, dass eine Zunahme an Einsicht und die zugehörige Fähigkeit zum Mentalisieren der eigenen Person und des Gegenübers zum Verständnis sowie der therapeutischen Klärung maladaptiver interpersoneller Muster führen können. Die Module VI bis VIII ermöglichen die Anwendung psychodynamischer Interventionen unter dem Blickwinkel unterschiedlicher Handlungsmodelle (• Tab. 1).

\section{Lernstrategien}

Entsprechend dem im Fokus stehenden Vorhaben der Vermittlung prozeduraler kommunikativer Kompetenzen wurde kongruent hierzu von der Steuerungsgruppe eine Methodik auf der Ebene "shows how" der Pyramide von Miller (1990) gewählt. Dies bedeutet, dass die therapeutischen Kompetenzen in einem geschützten, simulierten Setting vermittelt werden, im Sinne von „Als-ob“Situationen (Dieckmann et al. 2008). Die Definitionen von Rollenspielen und standardisierten Patienten (Barrows 1968) wurden in der Einleitung dieses Beitrags gegeben. Vorteile des Peer-Rollenspiels sind darin $\mathrm{zu}$ sehen, dass die Ausbildungsteilnehmer „ihre eigenen Patienten“ ins Seminar „mitbringen“, spielen und in der Rolle „ihrer Patienten" auch deren Innenperspektive einnehmen (Bosse et al. 2012) können. Außerdem erhalten sie Einschätzungen sowie Rückmeldungen der Seminarteilnehmer und des Dozenten zu „ihren Fällen“. Der Vorteil der standardisierten Patienten offenbart sich in dem Um- 
Beschwerden Niedergeschlagenheit, Freud- und Interesselosigkeit, sozialer Rückzug, Antriebslosigkeit. innere Unruhe und Versagensängste; Durchschlafstörungen und morgendliches Früherwachen

Aktuelle Als Architekt sei er bislang nur planerisch tätig gewesen, durch eine Vertretungssituation nun jedoch gezwungen, eine Bauleitung zu Auslöser übernehmen. Auf der Baustelle sei es zu Auseinandersetzungen mit den Handwerkern gekommen. Er habe sich zwischen den widersprüchlichen Interessen des Bauherrn und der Dienstleister aufgerieben. Krankschreibung vor 3 Monaten

Biografie Seine Mutter (+26 Jahre; Friseurin) habe 4 Kinder geboren, alle Geschwister seien Frühgeburten gewesen und verstorben. Er habe es als einziger im Brutkasten "geschafft". Trennung seiner Eltern im 4. Lebensjahr. Er sei bei den Großeltern väterlicherseits und beim Vater (+26 Jahre; Druckereimeister) aufgewachsen. Die Mutter habe er 14-tägig am Wochenende besucht. Nach erneuter Heirat des Vaters in seinem 8. Lebensjahr habe er mit ihm, der Stiefmutter und 3 jüngeren Stiefgeschwistern unter einem Dach gelebt. Er habe sich „zu keiner Familie richtig zugehörig" gefühlt. In seiner ersten Psychotherapie während des Studiums sei der Wunsch nach Kontakt zur Mutter aufgekommen, was das Verhältnis zum Vater belastet habe. Seine Mutter habe versucht, inn in ihre neue Familie zu integrieren. Im Kontakt mit ihr seien Engegefühle entstanden, weshalb er sich wieder distanziert habe. Bis heute bestünde der Wunsch nach einem besseren Verhältnis zu ihr; aktuell gebe es keinen Kontakt. Während der Schulzeit habe er unter Versagens- und Prüfungsängsten gelitten. Sein Architekturstudium habe er zeitweise für ein Zahnmedizinstudium unterbrochen. Das Ende des Architekturstudiums habe er sehr genossen, da er seine Leidenschaft zur Häuserplanung entdeckt habe. Bei solch kreativen Tätigkeiten habe er sich stets sehr lebendig gefühlt. Anschließend habe er in einem Architekturbüro von einem seiner ehemaligen Professoren, einer "Vaterfigur" für ihn, gearbeitet. Nach dessen Ausscheiden Tätigkeit in verschiedenen Architekturbüros, zuletzt sei er von einem renommierten Architekten abgeworben worden

Soziale Sein aktueller Chef habe auch eine „väterliche Funktion“ für ihn. Eine Teilhaberschaft an der Firma sei bereits in die Wege geleitet worSituation den. Nach seinem mehrwöchigen Arbeitsausfall befinde er sich aktuell in einem Wiedereingliederungsverfahren. Letzte Partnerschaft vor 11 Jahren. Vergangenes Jahr habe er sich zu einer verheirateten „Bauherrin“ hingezogen gefühlt, wobei diese ebenfalls seine Nähe gesucht habe. Zu einer Beziehung oder sexuellen Kontakten sei es jedoch nicht gekommen. Die erste „richtige Beziehung“ für 1,5 Jahre habe er im Alter von 18 Jahren gehabt, was eine „intensive Erfahrung" gewesen sei. Jedoch habe er das Gefühl gehabt, weder "mit ihr noch ohne sie leben zu können“. Vor 2 Monaten habe es eine neue Bekanntschaft gegeben, die sich wieder verlaufen habe. Er pflege freundschaftliche Kontakte, auch zu Personen aus einer zurückliegenden Gruppentherapie

Kranken- Anamnestisch weiter bekannt:Z.n. Tonsillektomie, beidseitiger Astigmatismus, Migräne mit Aura. Aktuelle Medikation: Amitriptylin geschichte $\quad\left(\right.$ Saroten $\left.^{\circledR}\right) 25 \mathrm{mg}(0-0-1)$ zur Schlafregulation. Psychotherapeutische Vorerfahrung: Zu Beginn des Studiums psychotherapeutische ambulante Einzeltherapie über ca. 20 Sitzungen; im Verlauf des Studiums 2,5 Jahre Gruppenpsychotherapie bei 2 Therapeuten

Psychischer Herr S. kleidet sich unauffällig, imponiert angepasst und unsicher. Im Kontakt dominiert der Leitaffekt der Melancholie. Er wirkt geBefund quält bei dem Versuch zu erspüren, was seine „eigenen“ Affekte sind. In der Gegenübertragung löst er Interesse und Sympathie aus. Die Arbeit mit ihm ist aufgrund seiner schnellen Auffassungsgabe und selbstreflexiven Kompetenzen anregend. Abwehrmechanismen sind Idealisierung, Rationalisierung, Verdrängung und Selbstentwertung

Psycho- $\quad$ Es bestehen ein ödipaler Konflikt im passiven Modus sowie eine narzisstische Tönung auf dem Boden einer depressiv-masochistischen dynamische Persönlichkeitsstruktur. Nach OPD liegt ein mäßig integriertes Strukturniveau vor, mit Schwierigkeiten in der Affektdifferenzierung, Einordnung Selbstwertregulation und Kommunikation nach innen. Sein Beziehungsverhalten ist durch das Bemühen gekennzeichnet, den Erwartungen des Gegenübers gerecht zu werden, um dem (fantasierten) Objektverlust zuvorzukommen. Zeitgleich wird die quälende Unsicherheit, möglicherweise nicht zu genügen, deutlich spürbar. Dieses Empfinden mündet in den Versuch, das Gegenüber unter keinen Umständen zu verlieren und hierfür nötigenfalls alles zu opfern. Beim Versagen dieses Kompensationsversuchs kommt es zum depressiven Einbruch

OPD Operationalisierte Psychodynamische Diagnostik

stand, dass diese dem Rollenspiel häufig einen größeren Realitätsgrad einer klinischen Situation verleihen können als im Peer-Rollenspiel. Sie ermöglichen es, den Handlungsrahmen des Rollenspiels besser aufrechtzuhalten, womit auch komplexere klinische/therapeutische Situationen darstellbar sind, und können ein strukturiertes, professionelles Feedback vermitteln (Schultz et al. 2018). Vergleichende Studien aus der Medizinerausbildung zum Training mit PeerRollenspielen einerseits und standardisierten Patienten andererseits liefern im Hinblick auf die erworbenen kommunikativen Kompetenzen weitgehend äquivalente Ergebnisse (Lane und Rollnick
2007). Um die jeweiligen Vorteile beider Methoden zu integrieren, wurde bei der Konzeption des Curriculums sowohl der Einbezug von Peer-Rollenspielen als auch von standardisierten Patienten vorgesehen. Neben dem Feedback von Peers, standardisierten Patienten und Dozenten werden den Teilnehmern die für die Evaluation (s. Abschn. „E Implementierung“) gewonnenen Daten der Prä-post-Selbsteinschätzung als auch die Prä-post-Videofilme personifiziert im Sinne eines digitalen Portfolios auf einem geschützten, personifizierten Laufwerk zur Verfügung gestellt.

Zur Vorbereitung der von den Peers einzubringenden Rollenspiele erhalten die Ausbildungsteilnehmer 3 bis 4 Tage vor dem Seminar eine Instruktion, in der sie darum gebeten werden, aus ihrer klinischen Arbeit heraus einen Patientenfall „mitzubringen“, anhand dessen sich die Intervention des Moduls möglicherweise gut üben lässt. Für das Modul der Widerstandsdeutung wäre dies beispielsweise ein Patientenfall, bei dem die Ausbildungsteilnehmer in ihrer Arbeit mit dem Patienten Widerstände ganz greifbar erlebt haben oder das Gefühl hatten, mit ihm „auf der Stelle zu treten“. Die Patientenfälle, die von den standardisierten Patienten verkörpert werden, folgen in ihrer Konzeption dem Ansatz von Schultz et al. (2018) sowie 
Tab. 3 Ablauf eines Moduls des DYNAMIK-Curriculums

\begin{tabular}{|c|c|c|}
\hline \multirow[t]{7}{*}{ Mittwoch } & $20.00-20.20 \mathrm{Uhr}$ & Begrüßung und Ablauf (Plenum) \\
\hline & \multirow[t]{3}{*}{$20.20-21.00 \mathrm{Uhr}$} & $\begin{array}{l}\text { Prä-Assessment: Erfassung der Ausgangskompetenzen, } \\
\text { Selbsteinschätzung }\end{array}$ \\
\hline & & Videografiertes Rollenspiel der ATN mit SP \\
\hline & & Selbstreflexion in Fragebogen und Freitext \\
\hline & \multirow[t]{3}{*}{$21.00-22.00 \mathrm{Uhr}$} & Theoretischer Input und Lernen am Modell (Kleingruppe) \\
\hline & & Vortrag des Modulleiters \\
\hline & & Rollenspiel des Modulleiters mit SP vor dem Plenum \\
\hline \multirow[t]{5}{*}{ Freitag } & \multirow[t]{5}{*}{ 18.30-21.30 Uhr } & Übung: videografierte Peer-Rollenspiele (Kleingruppe) \\
\hline & & Spielen „eigener“ Fälle aus klinischer Arbeit der ATN \\
\hline & & $\begin{array}{l}\text { ATN nehmen je Rolle des Therapeuten/Patienten/Feedbackgebers } \\
\text { ein }\end{array}$ \\
\hline & & $\begin{array}{l}\text { Selbstreflexion des Spiels am Video, zeitgleich Erarbeitung des } \\
\text { Gruppenfeedbacks }\end{array}$ \\
\hline & & 7 min Spiel, 7 min Reflexion, 15 min Gruppenfeedback \\
\hline \multirow[t]{9}{*}{ Samstag } & \multirow[t]{5}{*}{ 09.30-14.00 Uhr } & Übung: videografierte Rollenspiele mit SP (Kleingruppe) \\
\hline & & SP verkörpert 7 Sitzungsszenen über den Therapieverlauf \\
\hline & & ATN nehmen je Rolle des Therapeuten/Feedbackgebers ein \\
\hline & & $\begin{array}{l}\text { Selbstreflexion des Spiels am Video, zeitgleich Erarbeitung des } \\
\text { Gruppenfeedbacks }\end{array}$ \\
\hline & & 7 min Spiel, 7 min Reflexion, 15 min Gruppenfeedback \\
\hline & \multirow[t]{3}{*}{$14.00-15.00 \mathrm{Uhr}$} & $\begin{array}{l}\text { Post-Assessment: Erfassung der Kompetenzentwicklung, } \\
\text { Selbsteinschätzung }\end{array}$ \\
\hline & & Videografiertes Rollenspiel der ATN mit SP \\
\hline & & Selbstreflexion in Fragebogen und Freitext \\
\hline & $15.00-15.30 \mathrm{Uhr}$ & Offene Fragen und Resümee (Plenum) \\
\hline
\end{tabular}

Tab. 4 Vignette als Einstieg in ein Rollenspiel mit Ausbildungsteilnehmer und standardisiertem Patienten aus dem Modul „Basisinterventionen im Kontext von Übertragung und Gegenübertragung"

(Standardisier- Vergangene Woche standen Sie bei ihrem Psychotherapeuten vor verschlosseter) Patient

Therapeut ner Türe. Als Sie bei inm anriefen, hörten Sie auf dem Anrufbeantworter, dass er diese Woche im Urlaub sei. Sie können sich jedoch nicht erinnern, dass er dies im Vorfeld mit Ihnen besprochen hätte. Insgesamt war das sehr ungünstig für Sie, da Sie dringenden Gesprächsbedarf verspürten. In der heutigen Sitzung sprechen Sie dies nun an, allerdings sehr vorsichtig und ohne jeglichen Ärger zu zeigen; außerdem nehmen Sie die Schuld direkt auf sich selbst, wahrscheinlich sei Ihnen diese Information einfach entgangen. Sie begrüßen ihn mit den Worten: „Heute sind Sie also da."

Bitte schweigen Sie im Anschluss und warten Sie eine Intervention Ihres Therapeuten $a b$

Sie erwarten Herrn S. zur 35. Sitzung Ihrer ambulanten Einzelpsychotherapie, nachdem Sie eine Woche im Urlaub waren. Herr S. erscheint völlig entgegen seiner Gepflogenheiten 5 Minuten zu spät zur Stunde. Er wirkt auf Sie vordergründig freundlich, gleichzeitig jedoch unter Druck, meidet direkten Blickkontakt. Ihr Patient wird das Gespräch eröffnen. Bitte reagieren Sie darauf und greifen insbesondere auf die Interventionstechnik des Konfrontierens zurück. Das Gespräch soll einen Therapieausschnitt von 7 Minuten repräsentieren

Schauenburg et al. (2020; - Tab. 2). Hervorzuheben ist, dass die Rollenskripte der standardisierten Patienten Szenen umfassen, die sich vom Beginn bis zum Ende der ambulanten psychotherapeutischen Behandlung erstrecken. So kann

\section{E Implementierung}

Die Steuerungsgruppe eruierte zusammen mit dem Leitungsgremium die mögliche zeitlich-strukturelle Integration der 8 Module in das Ausbildungscurriculum des HIP (Schauenburg et al. 2019). Es wurde festgelegt, dass pro Ausbildungsjahr 2 der 8 Module stattfinden sollen. Ein Modul wird jeweils durch einen Modulleiter und 3 Kleingruppenleiter mit Erfahrung im jeweiligen Interventionsbereich geleitet. Ein Modul gliedert sich in einen Einführungsabend (Teil I) und ein Wochenendseminar (Teile II und III), deren zeitliche Abläufe in - Tab. 3 dargestellt und deren Inhalte im Folgenden skizziert werden.

\section{Einführungsabend (Teil I; 2 Unterrichts-} einheiten [UE]). Der Dozent und Modulleiter vermittelt das theoretische Wissen zu den in diesem Modul behandelten Interventionen anhand eines Vortrags und der Literatur. Daraufhin illustriert er die Interventionen als Rollenmodell in direkter Interaktion mit einem standardisierten Patienten vor der Ausbildungsgruppe. Im Plenum wird das Vermittelte und Erfahrene reflektiert.

Wochenendseminar, Freitag (Teil 2; 4 UE). Die Ausbildungsteilnehmer erproben und reflektieren ihre innere Haltung und die zu lernenden Interventionen in 7-minütigen Rollenspielen mit ihren Peers in Kleingruppen $(n=5)$ im geschützten Rahmen. Sie nehmen die Perspektive des Patienten, des Therapeuten oder eines beobachtenden Dritten ein. Die Übungen werden jeweils videografiert. Feedback erhalten sie über die Sichtung der Videoaufnahmen und von ihren Peers, moderiert durch den Seminarleiter. Dieser fasst die „teachable moments" der Übungssequenz zum Abschluss zusammen und erweitert um fehlende Aspekte.

Wochenendseminar, Samstag (Teil 3; 6 UE). In Kleingruppen üben die Ausbildungsteilnehmer die Interventionen mit einem standardisierten Patienten. Die Szenen stellen jeweils Ausschnitte aus psychotherapeutischen Sitzungen dar, die den zeitlichen Bogen vom Beginn 
Tab. 5 Ablauf der Kleingruppenarbeit und Feedbackalgorithmus (modifiziert nach Schultz et al. 2018)

(2)

bis zum Ende der ambulanten psychotherapeutischen Behandlung umfassen. Ein Beispiel der Instruktion für den standardisierten Patienten und den Teilnehmer, der die Rolle des Therapeuten übernimmt, zeigt • Tab. 4. Die Übungen werden jeweils videografiert. Nach ihrer Übungseinheit reflektieren die Ausbildungsteilnehmer ihr Rollenspiel jeweils zunächst selbst anhand des Videos, erhalten dann das Feedback des standardisierten Patienten und schließlich Rückmeldungen der Kleingruppe, moderiert durch den Seminarleiter. Feedback ist als eine „differenzierte Rückmeldung und Information zum Vergleich eines zu beobachtenden Verhaltens eines Ausbildungsteilnehmers mit einem erwünschten Qualitätsstandard zu verstehen, mit der Intention, die Handlungskompetenz der Lernenden zu verbessern" (van de Ridder et al. 2015). Feedbackprozesse stellen generell einen relevanten Faktor für das Lernen in ganz unterschiedlichen Kontexten dar (Ehrenthal et al. 2019). Zum Abschluss der Feedbacksequenz fasst der Dozent die „teachable moments" der Übungssequenz zusammen und erweitert diese um fehlende Aspek- te. Der Ablauf der Kleingruppenarbeit und der Feedbackalgorithmus sind in - Tab. 5 illustriert.

\section{F Evaluation}

Für die Bewertung der einzelnen Module ist eine Mehrebenenevaluation vorgesehen, die die im Folgenden beschriebenen Aspekte umschließt.

Akzeptanz der Module und Modulelemente. Von den Teilnehmern werden der Theorievortrag, das Rollenspiel des Dozenten, das Training mit den PeerRollenspielen und standardisierten $\mathrm{Pa}$ tienten, das Videofeedback und das Feedback der Peers, der standardisierten Patienten und der Dozenten auf LikertSkalen bewertet. Im Freitext schildern sie ihre zentralen persönlichen Lernerfahrungen und Erkenntnisse dieses Moduls.

Erfassung des subjektiv empfundenen Kompetenzzuwachses und Transferpotenzials. Die Selbsteinschätzung der Interventionskompetenz werden vor und nach dem jeweiligen Modul im Hinblick auf theoretische Inhalte, den subjektiv erlebten Kompetenzzuwachs und die wahrgenommene Fähigkeit, die erlernten Aspekte im Transfer in den eigenen Patientenbehandlungen anzubringen, auf Likert-Skalen erfasst.

Videoevaluation. Die Teilnehmer werden jeweils vor und nach einem Modul in Rollenspielen von standardisierten Szenen mit standardisierten Patienten videografiert, in denen sie die Aufgabe haben, einer Instruktion folgend, die Intervention, die jeweils Thema des Moduls ist, im Rollenspiel zu demonstrieren. Die gefilmte Episode dient sowohl einem Prä-post-Assessment als auch als einem persönlichen Lernportfolio der Teilnehmer. Die Videos des Prä-postAssessments werden nach Abschluss der Implementierung von Ratern, die hinsichtlich des Messzeitpunkts verblindet sind, anhand von Checklisten $\mathrm{zu}$ inhaltlicher, sprachlicher sowie patientenund situationsbezogener Umsetzung der Intervention (interventionsspezifische Checkliste) und globalen Rating-Skalen zur Kommunikation beurteilt (Nikendei et al. 2019; Wass et al. 2001).

\section{Befragung der Teilnehmer im Rahmen von halbstandardisierten Interviews.}

Die Teilnehmer werden mithilfe halbstandardisierter „Face-to-face“- und Telefoninterviews zu Aspekten von Inhalt, Methodik und Transfer in die eigenen Patientenbehandlungen befragt und die Transkripte inhaltsanalytisch ausgewertet.

\section{Diskussion und Ausblick}

Zielsetzung der vorliegenden Arbeit war die Darstellung der Konzeption, Gestaltung und Implementierung eines longitudinalen Curriculums zur Vermittlung psychodynamischer Interventionskompetenzen in der Ausbildung der tiefenpsychologisch-fundierten Psychotherapie. Im neuen Psychotherapiestudium an den psychologischen Instituten der Universitäten wird der praxisorientierte und kompetenzbasierte Unterricht in den anerkannten Methoden und Verfahren eine zentrale Rolle spielen. Hier wird einer frühen, longitu- 
dinalen und spiralförmigen Integration handlungsorientierter Lehrformen eine große Bedeutung zukommen. Bei der Entwicklung und Implementierung des vorgestellten DYNAMIK-Curriculums wurde sich an den Schritten des KernZyklus orientiert.

Zentrale didaktische Elemente dieses Curriculums sind die Rollenspiele mit Peers und standardisierten Patienten. Solche Simulationsmethoden bieten eine geschützte Umgebung, innerhalb derer therapeutische Interventionen „fehlerverzeihend" unter Berücksichtigung von Übertragungs- und Gegenübertragungsaspekten erprobt werden können. Insofern ist es sinnvoll - wie im DYNAMIKCurriculum konzeptionell verankert -, diese Lehr- und Lernmethoden bereits vor der Zwischenprüfung und damit vor der eigentlichen Behandlung ambulanter Psychotherapiepatienten einzusetzen. Das Peer-Rollenspiel ist die „kostengünstigere“ und „unaufwendiger verfügbare Methode“ (Bosse et al. 2012), die ein tiefgreifendes Verstehen der Patientensituation und -wahrnehmung sowie der Patient-Therapeut-Beziehung ermöglicht. Allerdings induziert das Peer-Rollenspiel bei den am Rollenspiel aktiv Beteiligten eher eine „unnatürliche" Bandbreite der Rolle (Dieckmann et al. 2008). So kann es z. B. zur gehäuften Darstellung sehr schwieriger, im Alltag jedoch selten anzutreffender Therapiesituationen kommen, die den Übungscharakter erschweren können. Standardisierte Patienten hingegen stabilisieren den Handlungsrahmen und ermöglichen damit auch die Umsetzung klinisch komplexer Szenarien. Es ist sicherlich notwendig, die differenziellen Vor- und Nachteile sowie spezifischen (zeitlichen) Einsatzmöglichkeiten von Peer-Rollenspielen und Rollenspielen mit standardisierten Patienten in der psychotherapeutischen Ausbildung weiter zu beleuchten. Sie sollten zukünftig einen Fokus der Psychotherapieausbildungsforschung bilden.

Den eigentlichen Wirkfaktor und das handlungsmodifizierende Agens stellt jedoch das Element des Feedbacks durch Peers, Dozenten und Video dar. Feedback umfasst die Bestandteile „Beobachtung“, „Bewertung“, „Anleitung“ und „Unter- stützung“ (Tuma und Nassar 2020). Qualitativ hochwertiges Feedback ist lernerzentriert sowie dialog- und diskussionsbasiert. Im Fokus stehen die vom Lernenden eingebrachten Aspekte, wobei es sich anbietet, mit positiven Aspekten, die dem Lernenden aufgefallen sind, zu beginnen, ehe zu kritischen Aspekten übergegangen wird (Pendleton et al. 2003). Feedback regt fokussierte, selbstreflexive Prozesse und die Auseinandersetzung mit dem eigenen therapeutischen Handeln an. Von Bedeutung ist die Einhaltung von „Feedbackregeln“ aller am Feedbackprozess Beteiligten, denen die Feedbackkriterien vermittelt werden müssen, da nur ein wertschätzendes Feedback Verhaltensänderungen anstoßen kann.

Unabhängig von der Therapieschule zeigen Teilnehmer in der Ausbildung zum psychologischen Psychotherapeuten einen ausgeprägten Wunsch nach engmaschigem und kontinuierlichem Feedback, um individuelle Muster und Schwächen frühzeitig zu erkennen und die Entwicklung eigener therapeutischen Fähigkeiten zu fördern. Feedback entfaltet insbesondere im Zusammenhang mit dem wiederholten, korrigierenden Üben seine Wirksamkeit („deliberate practice“; Ericsson et al. 1993). Allerdings ist $\mathrm{zu}$ beachten, dass die Wirkung von Feedback von einer Reihe unterschiedlicher Faktoren abhängt, wie z.B. von Form und Inhalt der Rückmeldung, von der Art und Komplexität der zu leistenden Aufgabe, der Beobachtungsqualität des Feedbackgebers und der (subjektiven) Wahrnehmung des Feedbackempfängers (van de Ridder et al. 2015).

Im Rahmen des DYNAMIK-Curriculums wird neben dem Peer-, Schauspieler- und Dozentenfeedback auch das Videofeedback zum Einsatz gebracht, das im Vergleich zu „herkömmlichem“ Feedback den Vorteil hat, dass sich Lernende aus einer Metaebene selbst beobachten und damit das eigene Handeln selbstreflexiv evaluieren können (Ozcakar et al. 2009). Im Hinblick auf die Einsatzmöglichkeiten und Wirkungsweise der verschiedenen Feedbackformate existieren bisher innerhalb der Psychotherapieausbildungsforschung keine differenziellen Erkenntnisse; auch sie sollten daher Gegenstand zukünftiger Forschung sein.
Die Vermittlung handlungsorientierter psychotherapeutischer Kompetenzen erfordert zudem die Implementierung und Integration von Prüfungsformen, innerhalb derer das beobachtbare Verhalten evaluiert, beurteilt und bewertet werden kann. Im Sinne eines „constructive alignment" (Biggs und Tang 2007) sollte die Lehr-/Lerneinheit auf der korrespondierenden Stufe der sog. Miller-Pyramide geprüft werden. Dies bedeutet, dass Lehr- und Lernformen, die sich - wie das DYNAMIK-Curriculum - auf der Stufe des „shows how “ befinden, hier also therapeutisches Handeln in einem simulierten Rahmen in Annährung an die Realität demonstriert werden muss, auch auf der Stufe des „shows how“ geprüft werden sollte. Für eine Prüfung handlungsorientierter psychotherapeutischer Kompetenzen böte sich in Anlehnung an die in der Medizin weit verbreite Prüfungsform OSCE (Wass et al. 2001) entsprechend eine „objektive structured therapeutic examination“ (OSTE) an. Bei einer solchen OSTE würden die Ausbildungsteilnehmer in mehreren Prüfungsstationen mit definierten Aufgabenstellungen konfrontiert und anhand standardisierter Rating-Skalen evaluiert (Nikendei und Jünger 2006). Während die OSCE an medizinischen Fakultäten in Deutschland weit verbreitet ist, existieren für die psychotherapeutische Ausbildung kaum Berichte über die Entwicklung und Implementierung eines solchen Prüfungsformats. Dies wäre aber neben der Leistungsevaluation von besonderer Relevanz, da handlungsorientierte Prüfungsformate das Lernverhalten im Sinne eines „assessment drives learning“ (Buss et al. 2012) positiv steuern.

\section{Fazit}

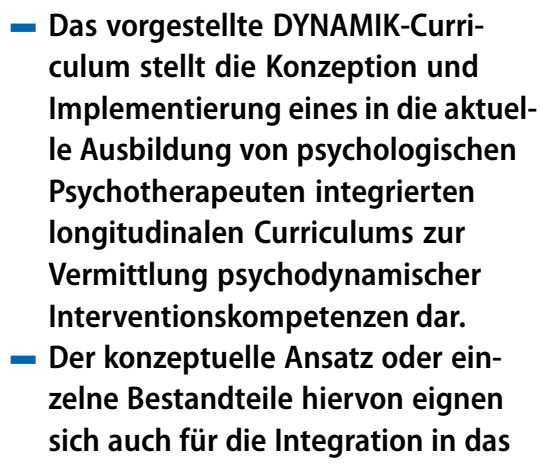


zukünftige „Psychotherapiestudium", die zukünftige Weiterbildung, institutsübergreifende Initiativen oder als einzelne Kursangebote an Ausbildungsinstituten.

- Es zeigt sich, dass solch eine longitudinale Verankerung möglich und umsetzbar ist. Herausforderungen sind im hohen personellen Aufwand, der v.a. der Kleingruppenarbeit geschuldet ist, und dem kostenintensiven Einsatz der standardisierten Patienten zu sehen.

- Zudem impliziert die Vermittlung psychotherapeutischer Interventionskompetenzen auch deren praktische Prüfung, die ebenfalls eines hohen Maßes an Logistik und finanzieller Aufwendungen bedarf.

- Zukünftige Forschungsfragen betreffen die Transferleistung der Lernerfahrungen eines solchen Curriculums in die Ausbildungstherapien und deren Einfluss auf das „Outcome“ dieser Therapien.

\section{Korrespondenzadresse}

\section{Prof. (apl.) Dr. med. C. Nikendei, MME} Zentrum für Psychosoziale Medizin, Klinik für Allgemeine Innere Medizin und Psychosomatik, Universitätsklinikum Heidelberg Thibautstr. 4, 69115 Heidelberg, Deutschland christoph.nikendei@med.uni-heidelberg.de

Förderung. Gefördert von der Köhler-Stiftung (D.10053237).

Funding. Open Access funding enabled and organized by Projekt DEAL.

\section{Einhaltung ethischer Richtlinien}

Interessenkonflikt. C. Nikendei, U. Dinger, J. Dück, J.C. Ehrenthal, T. Storck, S. Taubner, H.-C. Friederich, H. Schauenburg, J.-H. Schultz und I. Montan geben an, dass kein Interessenkonflikt besteht.

Die Ethikkommission der Medizinischen Fakultät der Universität Heidelberg erteilte ihre Zustimmung (S557/2019).

Open Access. Dieser Artikel wird unter der Creative Commons Namensnennung 4.0 International Lizenz veröffentlicht, welche die Nutzung, Vervielfältigung, Bearbeitung, Verbreitung und Wiedergabe in jeglichem Medium und Format erlaubt, sofern Sie den/die ursprünglichen Autor(en) und die Quelle ordnungsgemäß nennen, einen Link zur Creative Commons Lizenz beifügen und angeben, ob Änderungen vorgenommen wurden.

Die in diesem Artikel enthaltenen Bilder und sonstiges Drittmaterial unterliegen ebenfalls der genannten Creative Commons Lizenz, sofern sich aus der Abbildungslegende nichts anderes ergibt. Sofern das betreffende Material nicht unter der genannten Creative Commons Lizenz steht und die betreffende Handlung nicht nach gesetzlichen Vorschriften erlaubt ist, ist für die oben aufgeführten Weiterverwendungen des Materials die Einwilligung des jeweiligen Rechteinhabers einzuholen.

Weitere Details zur Lizenz entnehmen Sie bitte der Lizenzinformation auf http://creativecommons.org/ licenses/by/4.0/deed.de.

\section{Literatur}

Barrows HS (1968) Simulated patients in medica teaching. Can Med Assoc J 98:674-676

Biggs JB, Tang C (2007) Teaching for quality learning at university, 3. Aufl. McGraw-Hill, Maidenhead

Bosse HM, Schultz JH, Nickel M et al (2012) The effect of using standardized patients or peer role play on ratings of undergraduate communication training: a randomized controlled trial. Patient Educ Couns 87:300-306

Buss B, Krautter M, Möltner A et al (2012) Can the 'assessment drives learning' effect be detected in clinical skills training? Implications for curriculum design and resource planning. GMSZ Med Ausbild 29:Doc70

Crits-Christoph P, Gibbons MBC, Ternes CM et al (2010) Interpersonal accuracy of interventions and the outcome of cognitive and interpersonal therapies for depression. J Consult Clin Psychol 78:420-428

Dieckmann P, Rall M, Eich C et al (2008) Rollenspiel als wesentliches Element bei Simulationsverfahren in der Medizin. Z Evid Fortbild Qual Gesundhwes 102:642-647

Ehrenthal JC (2019) Erfahrungsbasiertes Lernen psychodynamischer Interventionen. Forum Psychoanal 35:413-428

Ehrenthal JC, Nikendei C, Huber J et al (2020) Prädiktoren des Lernerfolges eines erfahrungsbasierten Trainingsseminars zu psychodynamischen Interventionen. Verhaltenstherapie 30:128-136

Ehrenthal JC, Dinger U, Montan I, Nikendei C (2019) Neue Lehrformen zur Förderung therapeutischer Kompetenzen. Psychother Dialog 20:64-68

Ericsson KA, Krampe RT, Tesch-Römer C (1993) The role of deliberate practice in the acquisition of expert performance. Psychol Rev 100:363-406

Eubanks-Carter C, Muran JC, Safran JD (2015) Alliancefocused training. Psychotherapy 52:169-173

Fischer-Kern M, Doering S, Taubner S et al (2015) Transference-focused psychotherapy for borderline personality disorder: change in reflective function. Br JPsychiatry 207:173-174

Gumz A (2020) Umgang mit Spannungen und Krisen in der Therapiebeziehung. Psychotherapeut 65:119-132

Gumz A, Longley M, Schestag L, Hirschmeier C, Derwahl L, Weinreich J et al (2020) Die „Facilitative interpersonal skills"-Übung Messen therapeutischer Kompetenz mit der deutschsprachigen Version. Psychotherapeut 65:465-474
Gumz A, Rugenstein K, Munder T (2018) Allianz-Fokussiertes Training. Schulenübergreifender Weg zum Umgang mit Krisen in der therapeutischen Beziehung. Psychotherapeut 63:55-61

Hill CE (2014) Helping skills: facilitating exploration, insight, and action, 4. Aufl. American Psychological Association, Washington, DC

Hill CE, Lent RW (2006) A narrative and meta-analytic review of helping skills training: time to revive a dormant area of inquiry. Psychotherapy (Chic) 43:154-172

Hill CE, Sullivan C, Knox S et al (2007) Becoming psychotherapists: experiences of novice trainees in a beginning graduate class. Psychotherapy (Chic) 44:434-449

Horvath AO, Del Re AC, Fluckiger C et al (2011) Alliance in individual psychotherapy. Psychotherapy 48:9-16

Jennissen S, Huber J, Ehrenthal JC et al (2018) Association between insight and outcome of psychotherapy: systematic review and metaanalysis. Am J Psychiatry 175:961-969

Kern DE, Thomas PA, Howard DM et al (1998) Curriculum development for medical education. A six-step approach. Johns Hopkins University Press, Baltimore

Kühne F, Heinze PE, Weck F (2020) Standardized patients in psychotherapy training and clinical supervision: study protocol for a randomized controlled trial. Trials 21:276

Lane C, Rollnick S (2007) The use of simulated patients and role-play in communication skills training: a review of the literature to August 2005. Patient Educ Couns 67:13-20

Melluish S, Crossley J, Tweed A (2007) An evaluation of the use of simulated patient role-plays in the teaching and assessment of clinical consultation skills in clinical psychologists' training. Psychol Learn Teach 6:104-113

Miller GE (1990) The assessment of clinical skills/competence/performance. Acad Med 65:S63-S67

Nikendei C, Jünger J (2006) OSCE - praktische Tipps zur Implementierung einer klinisch-praktischen Prüfung. GMSZMed Ausbild 23:Doc47

Nikendei C, Bents H, Dinger U et al (2018) Erwartungen Psychologischer Psychotherapeuten zu Beginn ihrer Ausbildung: Qualitative Interviewstudie mit Vergleich von Verhaltens- und tiefenpsychologisch fundierter Psychotherapie. Psychotherapeut 63:445-457

Nikendei C, Huber J, Ehrenthal JC et al (2019) Intervention training using peer-role play and standardized patients in psychodynamic psychotherapy trainees. Couns Psychother Res 19:508-522

Ozcakar N, Mevsim V, Guldal D et al (2009) Is the use of videotape recording superior to verbal feedback alone in the teaching of clinical skills? BMC Public Health 9:474. https://doi.org/10.1186/ 1471-2458-9-474

Partschefeld E, Strauß B, Geyer M et al (2013) Simulationspatienten in der Psychotherapieausbildung. Psychotherapeut 58:438-445

Pendleton D, Schofield T, Tate P et al (2003) The new consultation: developing doctor-patient communication, 2. Aufl. Oxford University Press, Oxford

Van de Ridder JMM, McGaghie WC, Stokking KM et al (2015) Variables that affect the process and outcome of feedback, relevant for medical training: a meta-review. Med Educ 49:658-673

Safran J, Muran JC, Demaria A, Boutwell C, EubanksCarter C, Winston A (2014) Investigating 
the impact of alliance-focused training on interpersonal process and therapists' capacity for experiental reflection. Psychother Res 24:269-285

Safran JD, Muran JC, Eubanks-Carter C (2011) Repairing alliance ruptures. Psychotherapy 48:80-87

Schauenburg H, Dinger U, Kriebel A et al (2019) Zur Entwicklung tiefenpsychologischer Ausbildungsinstitute. Psychotherapeut 64:46-54

Schauenburg $H$, Jennissen S, Cierpka M (Hrsg) (2020) Heidelberger Standards zur psychodynamischen Diagnostik nach OPD-2. In: König K, Nikendei C, Tabatabai J (Hrsg) Heidelberger Standards, 1. Aufl. Medizinische Fakultät Heidelberg Heidelberg

Schultz JH, Alvarez S, Nikendei C (Hrsg) (2018) Heidelberger Standardgespräche, 1. Aufl. Medizinische Fakultät Heidelberg, Heidelberg

Sheen J, McGillivray J, Gurtman C, Moyd L (2015) Assessing the clinical competence of psychology students through objective strukctured clinical examinations (OSCEs): student and staff views. Aust Psychol 50:51-59

SteinertC, MunderT, Rabung Setal (2017) Psychodynamic therapy: As efficacious as other empirically supported treatments? A meta-analysis testing equivalence od outcomes. Am J Psychiatry 174:943-953

Strauß B, Barnow S, Brähler E et al (2009) Forschungsgutachten zur Ausbildung von Psychologischen PsychotherapeutInnen und Kinderund JugendlichenpsychotherapeutInnen. Im Auftrag des Bundesministeriums für Gesundheit. www.uniklinikum-jena.de/mpsy media/Downloads/Forschung/Endfassung_ Forschungsgutachten_Psychotherapieausbild ung.pdf.Zugegriffen: 19. Juni 2020

Taubner S, Kächele H, Visbeck A et al (2010) Therapeutic attitudes and practice patterns among psychotherapy trainees in Germany. Eur $J$ Psychother Couns 12:361-381

Tuma F, Nassar AK (2020) Feedback in medical education. StatPearls, Treasure Island

Wass V, Van der Vleuten C, Shatzer J et al (2001) Assessment of clinical competence. Lancet 357:945-949

Yap K, Bearman M, Thomas N, Hay M (2012) Clinical psychology students' experiences of a pilot objective structured clinical examination. Aust Psychol 47:165-173

\section{Märchenhafte Leichtigkeit}

Erste umfassende Studie zu Wohlfühlfilmen und ihrem Publikum

Sentimental und vermeintlich intellektuell anspruchslos - der Begriff »Wohlfühlfilm« wird in der Filmkritik meist abwertend verwendet. Demgegenüber spricht ein interessiertes Publikum, das Filme gezielt wegen ihres Wohlfühlfaktors anschaut, eher wohlwollend davon. Eine Studie am Max-Planck-Institut für empirische Ästhetik hat nun erstmals untersucht, welche Filme aus Zuschauersicht prototypische Wohlfühlfilme sind und was den Wohlfühlfaktor ausmacht.

Rund 450 Teilnehmerinnen und Teilnehmer aus Deutschland, Österreich und den deutschsprachigen Regionen Belgiens und der Schweiz nahmen an der Studie teil. Dabei zeigte sich, dass vor allem romantische Komödien ein besonders hohes Wohlfühlpotenzial besitzen. Als typische »Feel-good«Filme wurden unter anderem »Tatsächlich... Liebe«, »Pretty Woman«, »Die fabelhafte Welt der Amélie« und »Ziemlich beste Freunde» genannt.

Die Studienergebnisse liefern Anhaltspunkte dafür, welche inhaltlichen und formalen Eigenschaften Filme erfüllen müssen, damit sich das Publikum beim Anschauen besonders wohlfühlt: „Neben einer humoristischen Note und dem klassischen Happy End zeichnen sich Feel-good-Filme auch durch bestimmte wiederkehrende Handlungsmuster und Figuren aus", erklärt Studienleiter und Erstautor Keyvan Sarkhosh. „Oft sind es Außenseiter, die auf der Suche nach der wahren Liebe sind, die sich bewähren und gegen widrige Umstände kämpfen müssen und am Ende ihre Rolle in der Gemeinschaft finden."

\section{Wie setzt sich ein typischer} „Wohlfühlfim« zusammen?

Zu den charakteristischen Bestandteilen gehören nicht nur aus Romantik und Humor, sondern auch dramatische Momente. Diese berühren die Zuschauerinnen und Zuschauer meist emotional stark. Zugleich sind sie oftmals in ein märchenhaftes Setting eingebettet, das ebenfalls typisch für Feel-goodFilme ist und wesentlich zur wahrgenommenen Leichtigkeit beiträgt. Nicht zuletzt die Mischung all dieser Elemente macht einen Wohlfühlfilm aus.

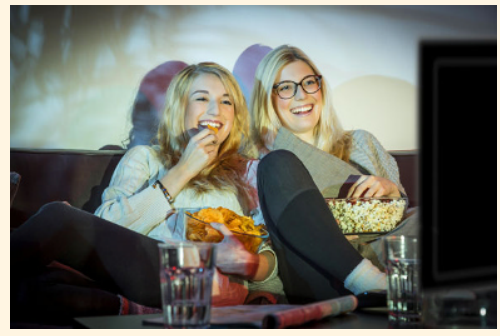

(c) tournee / Fotolia

Fazit

Die Studienergebnisse unterstreichen, dass sich viele Menschen Wohlfühlfilme gezielt anschauen, um zu entspannen und ihre Stimmung zu heben. Viele der Studienteilnehmerinnen und -teilnehmer stimmten darüber ein, dass Wohlfühlfilme zwar sentimental, aber nicht kitschig und vor allem handwerklich sehr gut gemacht sind. Hierin unterscheidet sich die positiv besetzte Verwendung des Labels aus Zuschauersicht sehr deutlich von der überwiegend negativen Haltung professioneller Filmkritikerinnen und Filmkritiker.

Die Ergebnisse der Studie wurden unlängst in der Fachzeitschrift Projections veröffentlicht.

Originalpublikation: Sarkhosh, K., und Menninghaus, W. (2021). The feelgood film: Genre features and emotional rewards. Projections, 15 (1), 55-92. DOI:10.3167/proj.2021.150104

Quelle: Max-Planck-Institut für empirische Ästhetik (www.ae.mpg.de

[29.04.2021]) 